\title{
Optimization of Complete Monopole Antennato Exhibit Wideband Capabilities.
}

\author{
ZahidGulzarKhaki ${ }^{1}$,Showkat Ahmad Dar ${ }^{2}$ \\ ${ }^{1}$ (Department of Electronics \& communication Engineering, Islamic University of Science and Technology, India) \\ ${ }^{2}$ (Department of Computer Science Engineering, Islamic University of Science and Technology, India)
}

\begin{abstract}
Antennas used for early portable wireless handheld devices were the so-called whip antennas. The quarter-wavelength whip antenna was very popular, mostly because it is simple and convenient. It has an Omnidirectional pattern in the plane of the earth when held upright and a gain satisfying the device's specifications. New antenna designs have appeared on radios with lower profile than the whip antenna and without significantly reducing performance. These include the quarter-wavelength helical antenna and the "stubby" helical antenna, which is the shortest antenna available. In recent years, the demand for compact handheld communication devices has grown significantly. Devices smaller than palm size have appeared in the market. Antenna size is a major factor that limits device miniaturization. In the past few years, new designs based on the Planar Inverted-F Antenna (PIFA) and Microstrip Antennas (MSA) have been popular for handheld wireless devices because these antennas have a low profile geometry instead of protruding as most antennas do on handheld radios. Conventional PIFAs and MSAs are compact, with a length that is approximately a quarter to a half of the wavelength. These antennas can be further optimized by adding new parameters in the design, such as strategically shaping the conductive plate, or judiciously locating loads.
\end{abstract}

Keywords-CPW,CST, PIFA, MSA,LAN, WiMAX

\section{Introduction}

The size of the antenna on the substrate should be very small. Conventional whip and helical antennas take large volume on the substrate. So in order to reduce the size of the antenna on the substrate, we use Micro strip antennas. The objective of designing the Monopole antenna is the miniaturization of antenna. Monopole antennas exhibit high gain and improve efficiency in a surprisingly small package. Monopole antenna can be designed to exhibit wideband capabilities.

\subsection{Design of simple $U$-shaped monopole antenna}

For the simple U-shaped antenna, it is seen that a first resonant mode at about $2.5 \mathrm{GHz}$ and a second mode at about $5.2 \mathrm{GHz}$ are obtained for the U-shaped monopole only. For the proposed antenna, the first resonant mode of the U-shaped monopole at about $2.5 \mathrm{GHz}$ is successfully excited with good impedance matching to cover the $2.4 \mathrm{GHz}$ WLAN and $2.5 \mathrm{GHz}$ Wi-MAX bands. The resonating path length of the Ushaped monopole is given as $L_{11}\left(L_{11}=\mathrm{S}+L_{3}+L_{1}\right)$. Thus the designed U-shaped monopole antenna is simulated using Computer Simulation Technology (CST) software.

\subsection{Design of simple Triangular-shaped monopole antenna}

For the simple Triangular shape antenna, the fundamental resonant mode is obtained at $3.2 \mathrm{GHz}$ [2]. Due to the electromagnetic coupling between the U-shaped and the Triangular shape monopole antenna, the fundamental resonant mode of the triangular monopole shift to $3.6 \mathrm{GHz}$, this also covers the WiMAX band at 3.5GHz.The resonating path length of the Triangular-shaped monopole is given as $L_{22}\left(L_{22}=\mathrm{S}+L_{4}+L_{2}\right)$. Thus the designed Triangular-shaped monopole antenna is simulated using CST software.

\subsection{Design of the monopole antenna by varying $P_{1}, P_{2}$ of the ground plane}

The monopole antenna is excited with a $50-\Omega$ CPW transmission line, having a signal strip of width $W_{1}$ and a gap of distance $g$ between the signal strip and the ground plane, is used. Two right triangles with legs $P_{1}, P_{2}$ on the coplanar waveguide (CPW) feeding mechanism are notched from the ground planes, which results in a smooth transition from one resonant mode to another and ensures good impedance match over a broad frequency range [3]. In addition, it should be noted that the ground plane dimensions can affect the resonant frequencies and operating bandwidths of the two operating bands. The effects of the parameters $P_{1}$ and $P_{2}$ of the bevelled ground plane on the impedance matching of the antenna, which have a small effects on the lower band. Conversely, the upper band is strongly affected by the variations in $P_{1}$ and $P_{2}$, and the impedance bandwidth is 
enhanced from $31 \%$ to $62 \%$ as $P_{1}$ increased from $5 \mathrm{~mm}$ to $9 \mathrm{~mm}$ and $P_{2}$ increased from $5 \mathrm{~mm}$ to $12 \mathrm{~mm}$. The results indicate that the impedance bandwidth of the upper band for the antenna can be independently controlled by adjusting the dimensions $P_{1}$ and $P_{2}$ of the ground plane.

\subsection{Design of the monopole antenna by varying the width $W_{2}$ of $U$-shaped strip}

The effect of varying the width $W_{2}$ of the U-shaped strip can be seen by the shift in the frequency of the simulated reflection coefficient graph. It is seen that, the first resonant mode of the U-shaped monopole is shifted to higher frequencies as $W_{2}$ increased, and the impedance bandwidth for the lower band is also enhanced. For this case, the antenna characteristic is similar to that of the rectangular monopole antenna .

\subsection{Design of the monopole antenna by varying the length $L_{1}$ of $U$-shaped strip}

The effect of varying the length $L_{1}$ of the U-shaped strip can be seen by using the simulated reflection coefficient. If the length $L_{1}$ of the U-shaped strip is varied from 15 to $21 \mathrm{~mm}$ by keeping the triangular patch length $L_{2}$ is fixed, the effects of the length $L_{1}$ on the impedance matching of the first and third resonant modes are seen to be larger than that on the second resonant mode. The results show that the lower and upper bands are shifted to lower frequencies, when the length $L_{1}$ is increased from 15 to $21 \mathrm{~mm}$.

\section{OptimizationOf Monopole Antenna}

\subsection{Simulation of the monopole antenna by varying $P_{1}, P_{2}$ of the ground plane}

By varying the parameters $P_{1}, P_{2}$ of the ground plane, there will be only small effect on the lower band. But the upper band is strongly affected, where the impedance bandwidth is improved.

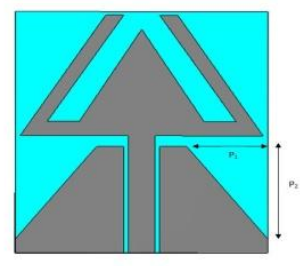

Fig.2.1: Structure of monopole antenna varying the parameters $P_{1}, P_{2}$

2.1.1 Results obtained for monopole antenna for different values of $P_{1}, P_{2}$

Return loss Vs Frequency

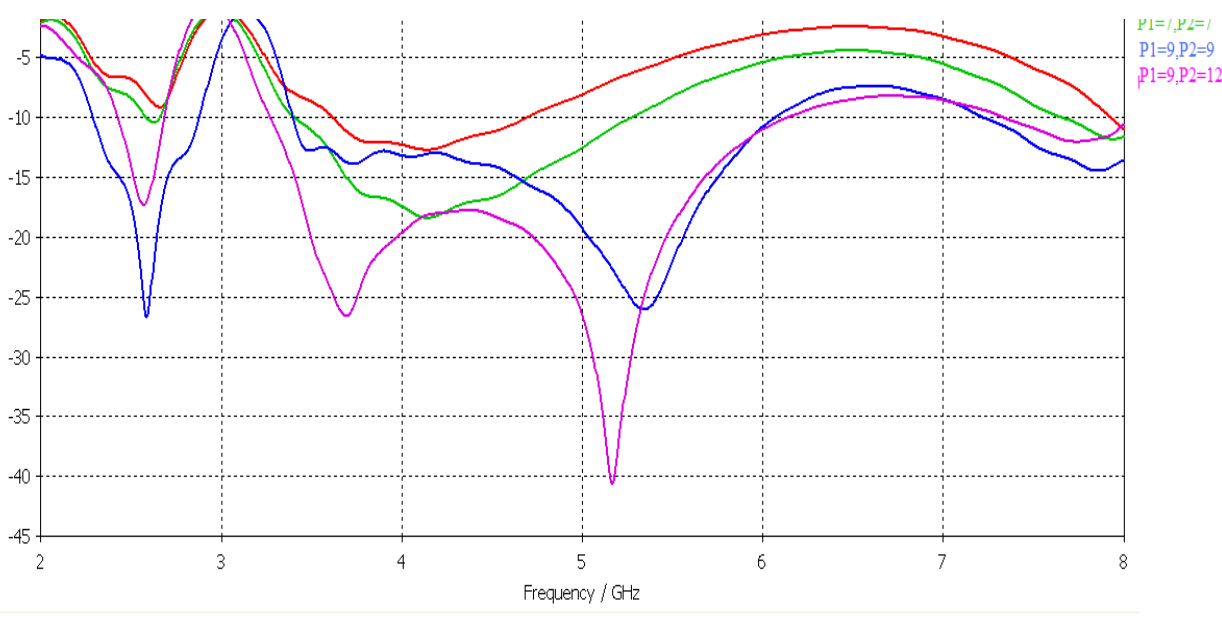

Fig 2.2: Return loss Vs Frequency plot 


\subsection{Simulation of the monopole antenna by varying the width $W_{2}$ of $U$-shaped strip}

By varying the width $W_{2}$ of the U-shaped monopole, the first resonant mode is shifted to the higher frequencies by which the impedance bandwidth of the lower band is enhanced and for higher band the bandwidth is affected slightly.

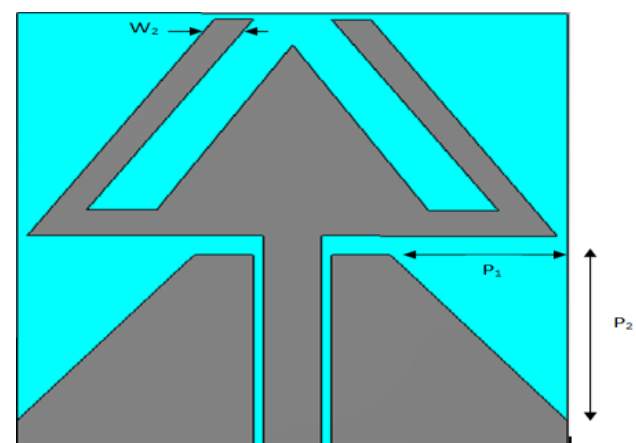

Fig.2.3: Structure of monopole antenna varying the parameters $W_{2}$

2.2.1 Results obtained for monopole antenna for different values of $W_{2}$ Return loss Vs Frequency

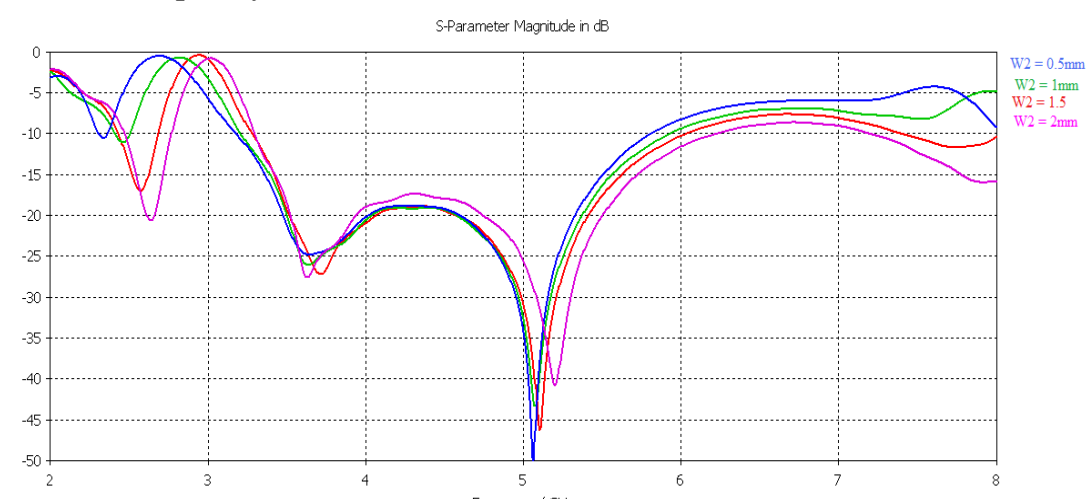

Fig 2.4: Return loss Vs Frequency plot

\subsection{Simulation of the monopole antenna by varying the length $L_{1}$ of $U$-shaped strip}

By varying the length $L_{1}$ of the U-shaped monopole, the lower and the upper bands are shifted to the lower frequencies. The impedance matching of the first and third resonant modes are seen to be larger than that of the second resonant mode.

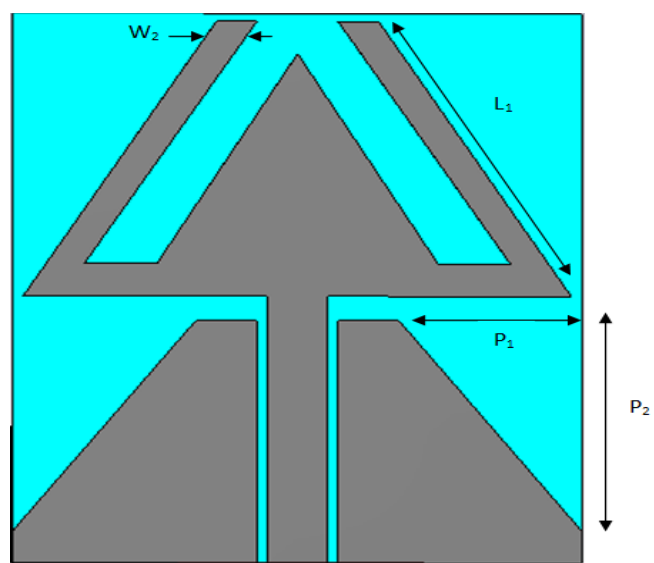

Fig.2.5: Structure of monopole antenna varying the parameters $L_{1}$ 
2.3.1 Results obtained for monopole antenna for different values of $L_{1}$ Return loss Vs Frequency

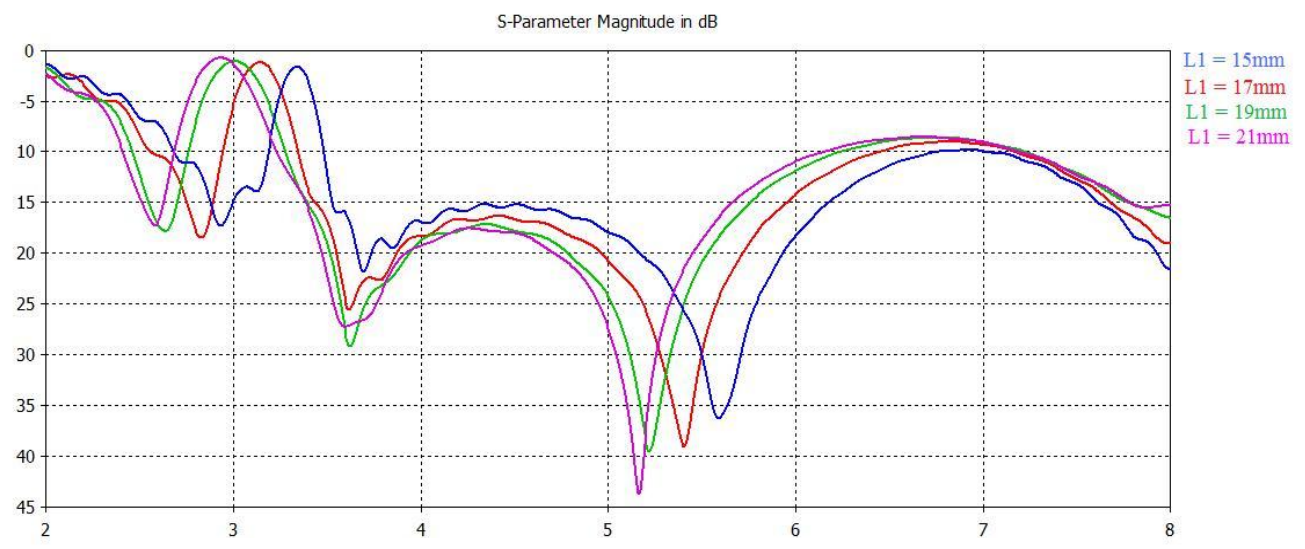

Fig 2.6: Return loss Vs Frequency plot

\section{Conclusion}

The CPW-feed monopole antenna with the assemble of monopoles is designed and simulated for the WLAN/WiMAX bands, ranging from 2.4 to $2.77 \mathrm{GHz}$ covering the standards of IEEE 802.11b/g. The antenna also covers the wideband of 3.2 to $6.3 \mathrm{GHz}$ for the standards of IEEE 802.11a, IEEE 802.16 including the mobile WiMAX applications. By shaping the ground plane elements we are getting the good impedance matching characteristics which can be operated for different wireless applications. The area occupied by this monopole antenna is about $28 \times 34 \mathrm{~mm}$ which is very much small in comparison with other basic antennas. The antenna shows the good dipole like radiations characteristics with the moderate gain over the operating bands which are attractive for practical applications in the communication gadgets. The radiation patterns are nearly Omni directional over the entire bandwidth.

\section{References}

[1] Constantine A. Ballanis "Antenna theory -analysis and design" second edition, John wiley\&son's .inc.

[2] Qing-Xin Chu and Liang-Hua Ye "Desig dual-wideband antenna for WLAN/WIMAX applications ," IEEE Trans.AntennaPropag., VOL. 58, NO. 12, Dec 2010

[3] Q. X. Chu and Y. Y. Yang, "A compact ultra wideband antenna with $3.4 / 5.5 \mathrm{GHz}$ dual band-notched characteristics," IEEE Trans.AntennaPropag., vol. 56, no. 12, pp. 3637-3644, 2008.

[4] H. D. Chen and H. T. Chen, "A CPW-fed dual-frequency monopole antenna," IEEE Trans.AntennaPropag., vol. 52, no. 4, pp.978982,Apr. 2004

[5] C. Y. Pan, T. S. Horng, W. S. Chen, and C. H. Huang, "Dual wideband printed monopole antenna for WLAN/WiMAX applications," IEEE Antennas Wireless Propag. Lett., vol. 6, pp.149-151, 2007.

[6] Design of Dual-band CPW-fed cross-slot monopole antenna for WLAN operation" was presented in IEEE Antennas Wireless Propag. Lett.,,vol,. 6, 2008.

[7] Qing-Xin Chu and Liang-Hua Ye "A compact dual-frequency antenna with triangular ring monopole and U-shaped sleeves", European microwave conference, 2009.

[8] Y.T. Lo, D. Solomon, “Theory and Experiment on Microstrip Antennas," IEEETrans. Ant. Prop., vol. AP-27, no. 2, March 1989, pp. $137-145$.

[9] L. Setian, "Practical Communication Antennas with Wireless Applications", Prentice Hall PTR, New Jersey: 1998.

[10] E.D. Caswell, W.A. Davis, and W.L. Stutzman, "Fundamental Limits on Antenna Size,” Artech House, Boston: 1992.

[11] Girish Kumar and K P Ray "Broadband micro strip antennas" by, Artech House antennas and propagation library. 